was not suppressed by these inhibitors (Figure 1e), indicating that the modulation of $\gamma$-secretase activity triggered by DDIA is not a downstream event of caspase cascades.

Because Bax can regulate apoptotic process in the upstream of caspase cascades, ${ }^{19}$ we examined whether $\gamma$ secretase activity is regulated by Bax translocation. When etoposide or camptothecin was added to $\mathrm{CHO}-\mathrm{C} 99$ cultures with/without furosemide (a Bax translocation inhibitor, $2 \mathrm{mM}$ ), the marked reductions in $\gamma$-secretase activity was observed in both cases (upper panel of Figure 1f).

Then, to determine whether $\mathrm{Bcl}-2 / \mathrm{Bax}$ system is essential for DDIA-elicited stimulation of $\gamma$-secretase, CHO-C99 cells were transiently transfected with Bcl-2 cDNA, which antagonizes Bax function. ${ }^{20}$ The overexpression of $\mathrm{Bcl}-2$ in $\mathrm{CHO}-\mathrm{C} 99$ cells dramatically blocked $\gamma$-secretase activation triggered by DDIAs (lower panel of Figure 1f), indicating that $\mathrm{Bcl}-2 / \mathrm{Bax}-d e p e n d e n t$ death pathway mediates the DDIAinduced modulation of $\gamma$-secretase activity.

Although previous reports suggest a close correlation between cell death and the $A \beta$-mediated pathogenesis of $A D$, few studies have demonstrated how cell death can affect the proteolytic processing of APP. Here, we provide evidence that DDIA-elicited $\gamma$-secretase activity is dependent on Bax/ $\mathrm{Bcl}-2$ pathway, but not on caspase cascades. Based on these results, we propose that cell death pathways including Bax translocation, triggered by various apoptotic stimuli, critically facilitate the generation of $\mathrm{A} \beta$ by activating $\gamma$-secretase. Because increased level of $A \beta$ acts as another death signal, a feedback loop between cell death and $A \beta$ generation can result in the progress of cell death process in the sporadic $A D$ brain. Our results suggest that blockade of apoptosis during the early pathologic stage presents as a good therapeutic target for the intervention in the pathogenesis of $A D$.
Acknowledgements. We thank Dr. Young-Keun Jung (Gwangju Institute of Science and Technology, Gwangju, Korea) for the C99-GV construct, Dr. Sangram Sisodia (University of Chicago) for the ANPP cell line and the $\triangle E N 1-G V$ construct, and Dr. Gyesoon Yoon (Ajou University, Suwon, Korea) for the Bcl-2 construct. This work was supported by KOSEF and AARC (RO1-2004-000-10271-0 and R11-2002-097-05001-2, respectively) and by the 21C frontier functional proteomics project of the Korean Ministry of Science and Technology (FPR05C2-010).

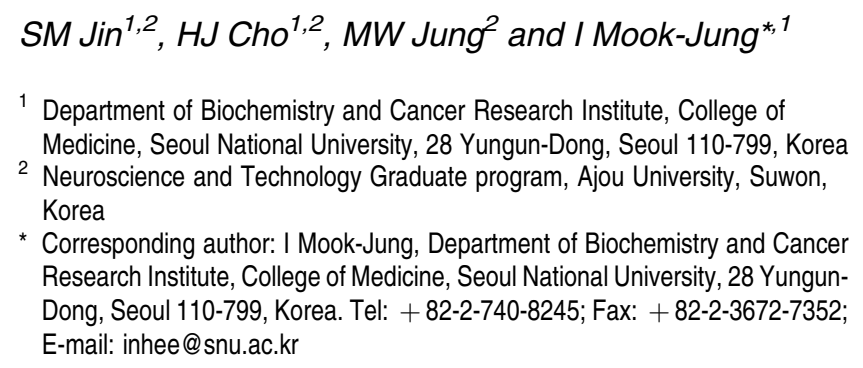

${ }^{1}$ Department of Biochemistry and Cancer Research Institute, College of Medicine, Seoul National University, 28 Yungun-Dong, Seoul 110-799, Korea

2 Neuroscience and Technology Graduate program, Ajou University, Suwon, Korea

* Corresponding author: I Mook-Jung, Department of Biochemistry and Cancer Research Institute, College of Medicine, Seoul National University, 28 YungunDong, Seoul 110-799, Korea. Tel: + 82-2-740-8245; Fax: + 82-2-3672-7352; E-mail: inhee@snu.ac.kr

1. Selkoe DJ. Physiol Rev 2001; 81: 741-766.

2. Esler WP, Wolfe MS. Science 2001; 293: 1449-1454.

3. Seubert $P$ et al. Nature 1993; 361: 260-263.

4. Wolfe MS, Haass C. J Biol Chem 2001; 276: 5413-5416.

5. Esler WP et al. Proc Natl Acad Sci USA 2002; 99: 2720-2725.

6. Yu G et al. Nature 2000; 407: 48-54.

7. Goutte $C$ et al. Proc Natl Acad Sci USA 2002; 99: 775-779.

8. Francis R et al. Dev Cell 2002; 3: 85-97.

9. Kimberly WT et al. Proc Natl Acad Sci USA 2003; 100: 6382-6387.

10. Edbauer D et al. Nat Cell Biol 2003; $5:$ 486-488.

11. Takasugi $\mathrm{N}$ et al. Nature 2003; 422: 438-441.

12. Galli $C$ et al. Proc Natl Acad Sci USA 1998; 95: 1247-1252.

13. Tesco G, Koh YH, Tanzi RE. J Biol Chem 2003; 278: 46074-46080.

14. Ohyagi $Y$ et al. Neuroreport 2000; 11: 167-171.

15. Kim S-K et al. FASEB J 2006; 20: 157-159.

16. Karlstrom $\mathrm{H}$ et al. J Biol Chem 2002; 277: 6763-6766.

17. Kim SH et al. J Biol Chem 2003; 278: 33992-34002.

18. Sawada M et al. Cell Death Differ 2000; 7: 761-772.

19. Cory S, Huang DC, Adams JM. Oncogene 2003; 22: 8590-8607.

20. Putcha GV, Deshmukh M, Johnson Jr EM. J Neurosci 1999; 19: 7476-7485.

\title{
Abrogation of signal-dependent activation of the cdk9/ cyclin T2a complex in human RD rhabdomyosarcoma cells
}

\author{
Cell Death and Differentiation (2007) 14, 192-195. doi:10.1038/sj.cdd.4402008; published online 14 July 2006
}

\begin{abstract}
Dear Editor,
During skeletal myogenesis, the transcription factor MyoD promotes differentiation in myoblasts by its ability to arrest the cell cycle and activate muscle-specific transcription. ${ }^{1,2} \mathrm{Re}-$ cently, we showed that the cdk9/cyclin T2a complex plays an essential role in the MyoD-dependent activation of the
\end{abstract}

myogenic program, describing a distinction with the cdk1 and cdk2 complexes, which are functionally downregulated during myotube formation and inhibit muscle-specific transcription when overexpressed. ${ }^{3}$ The role of cdk9 in modulating promoter-restricted transcription appears to be dependent 
on the sequence-specific factor recruiting the kinase, and on cdk9's ability to associate with different regulatory subunits, such as cyclin T1 and cyclin T2. ${ }^{4}$

In vitro, the cdk9/cyclin T2 complex immunoprecipitated from differentiating myoblasts can efficiently phosphorylate MyoD. ${ }^{3}$ To further investigate the relevance of this phosphorylation, we screened a panel of different MyoD point mutants where the serine of putative cdk target sites - proline-directed serine (sp sites) - were replaced by a nonphosphorylatable amino acid (alanine). We found that the purified His-MyoD mutant carrying the substitution of serine 37 (His-MyoDsp2) could not be phosphorylated by cdk9 (Figure 1a), indicating the phosphorylation of this residue as the possible signal enabling cdk9 to transactivate MyoD. To explore this possibility, we decided to compare the transcriptional activity of MyoDsp2 with two other point mutants carrying a mutation on sp sites targeted by cdk1 and cdk2, serine 5 (MyoDsp1) and serine 200 (MyoDsp3). During the G1 phase of the cell cycle, cdk2-dependent phosphorylation at serine 200 plays a crucial role in modulating MyoD half-life by triggering the proteosome-dependent degradation pathway. ${ }^{5-7}$ In late $G_{2}$, concomitant phosphorylation at serine 5 and serine 200 by cdk1 results in the decrease of MyoD activity and stability. In CH310T1/2 fibroblasts induced to differentiate MyoDwt and MyoDsp1 were efficiently transactivated by cdk9/cyclin T2a, whereas the activity of MyoDsp2 was even impaired by the cotransfection of cdk9/cyclin T2a (Figure 1c). We interpreted this effect as a result of the formation of a MyoD-cdk9/cyclin T2a a
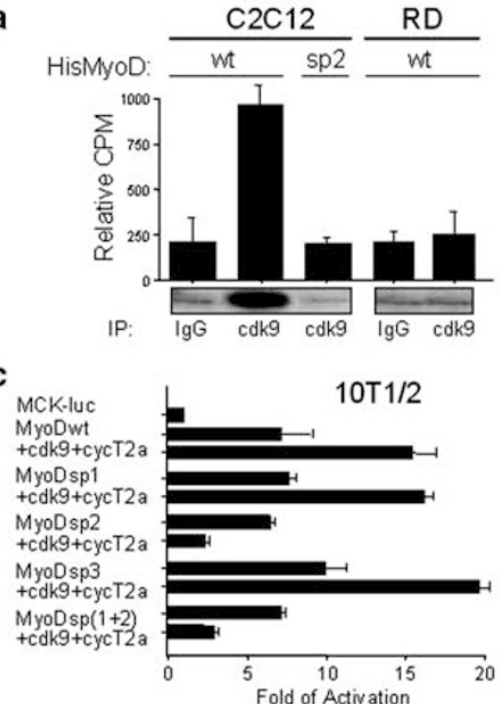

e

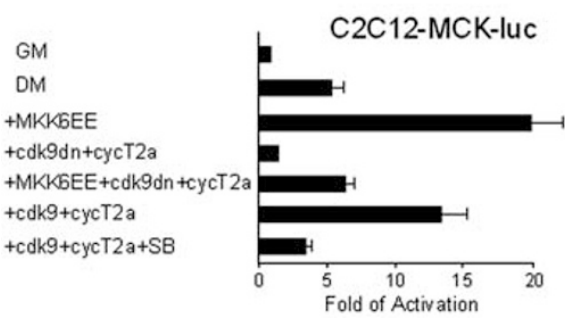

b

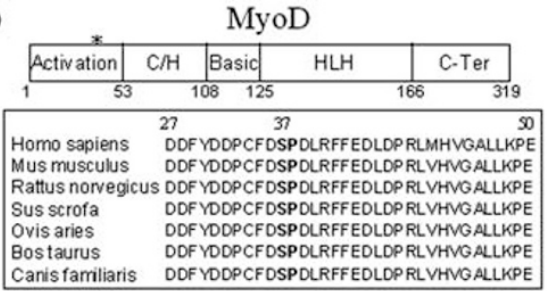

d

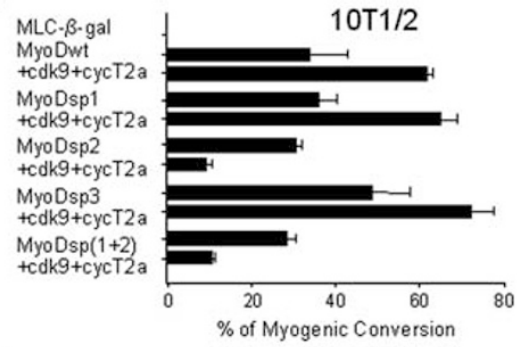

f

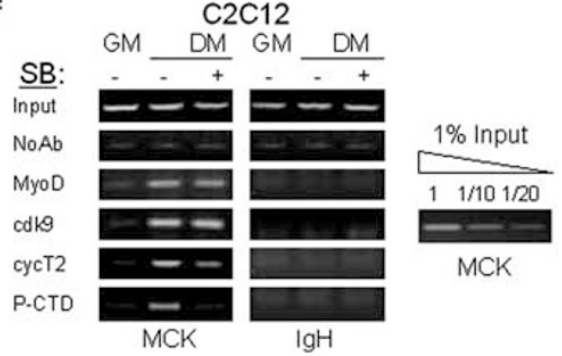

Figure 1 (a) Sequential kinase assay was performed as reported elsewhere. ${ }^{3,8}$ Briefly, C2C12 and RD cells were induced to differentiate by adding DMEM supplemented with $2 \% \mathrm{HS}(\mathrm{DM})$, and collected after $24 \mathrm{~h}$. The extracts were immunoprecipitated with affinity-purified anti-cdk9 and incubated with $1 \mu \mathrm{g}$ of dialyzed HisTag-mMyoDwt or HisTag-mMyoDsp2 in kinase reaction buffer. The samples were subjected to autoradiography and the quantitation of incorporated $\gamma^{32} \mathrm{P}$ was measured by scintillation counting. The indicated relative CPM levels were obtained by normalizing MyoD $\gamma^{32} \mathrm{P}$ incorporation on the densitometric evaluation of the amount of immunoprecipitated material (see Supplementary Information, C) and the abundance of substrate. (b) Schematic representation of MyoD structural domains and comparison of amino-acid sequences encompassing serine 37 in different species. (c) 10T1/2 fibroblasts were transfected in DMEM supplemented with 20\% FBS (GM), transferred in DM and harvested after $48 \mathrm{~h}$ as described previously. ${ }^{3}$ The luciferase assay was performed in triplicate and normalized by $\beta$-gal assay as described elsewhere. ${ }^{3}$ (Amount of DNA transfected: MCK-luc $0.3 \mu \mathrm{g}$; MyoDwt, sp1, sp2, sp3, sp $(1+2), 0.3 \mu \mathrm{g}$; cdk9 and cyclin T2a $0.5 \mu \mathrm{g} ; \beta$-gal $0.3 \mu \mathrm{g}$.) (d) Myogenic conversion assay was performed as described previously. ${ }^{3} \mathrm{MyoD}$ mutations were tested in their ability to convert fibroblasts either with or without co-transfection of cdk9/cyclin T2a. The cells were co-transfected with a reporter expressing $\beta$-galactosidase ( $\beta$-gal) fused to a nuclear localization signal and under the control of MLC promoter (MLC- $\beta$ gal), and the extent of myogenic conversion was scored by visualizing cells with more than one $\beta$-gal-positive nucleus. Seventy percent confluent $10 T 1 / 2$ cells were transfected with the indicated plasmids and induced to differentiate for $48 \mathrm{~h}$. Cells were fixed and stained following the $\beta$-gal standard protocol. Multinucleation was considered as a single cell with more than two $\beta$-gal-positive nuclei. (Amount of DNA transfected: MLC-gal $0.5 \mu \mathrm{g}$; MyoDsp1, sp2, sp3, sp(1+2) $0.5 \mu \mathrm{g}$; cdk9, cdk9dn and cyclin T2a $0.5 \mu \mathrm{g}$.) (e) $\mathrm{C} 2 \mathrm{C} 12$ polyclones containing integrated MCK-luc were generated as described. ${ }^{16}$ To evaluate luciferase activity, polyclones were transfected with MKK6EE, cdk9dn, cdk9 and cyclin T2a in various combinations as reported in the figure, transferred in DM and harvested after $48 \mathrm{~h}$. (Amount of DNA transfected: MKK6EE, cdk9dn, cycT2a $0.5 \mu \mathrm{g} ; \beta$-gal $0.3 \mu \mathrm{g}$. SB203580 $5 \mu \mathrm{M}$ (SB).) (f) ChIP assay was performed using the anti-cdk9 and anti-cyclin T2, ${ }^{3}$ together with the antiMyoD (Santa Cruz) and anti-P-RNAPollI H14 (Covance) antibodies according to Upstate Biotechnology instructions. PCR conditions were as described previously. ${ }^{14}$ SB: SB203580 $5 \mu \mathrm{M}$ 
complex that cannot be activated by cdk9 phosphorylation, thereby behaving as a dominant-negative toward uncomplexed MyoD. Transactivation of MyoDsp3 by cdk9/cyclin T2a was similar to that of MyoDwt and MyoDsp1. Finally, we analyzed the function of a MyoD mutant carrying both the mutations in Ser5 and in Ser37 (MyoDsp1+2). This construct behaved in the same way as MyoDsp2, confirming the possible implication of serine 37 in the transactivation of MyoD by cdk9/cyclin T2a. The same results were obtained by using the identical MyoD mutants fused to a Gal4 DNAbinding domain (Gal4MyoDwt, sp1, sp2 and sp3) with a Gal4-luciferase construct as a reporter (Supplementary Information, A). As we described previously, the MyoDNter does not interact with $\operatorname{cdk} 9,{ }^{3}$ but includes serine 37 . The fact that this mutant is not transactivated by cdk9/cyclin T2a suggests that two regions of $M y o D$ are required for full activation, the bHLH and serine 37 in the N-terminal transactivation domain. Interestingly, this finding seems to be in accordance with cdk9/cyclin T2's requirement of two different substrate subdomains, the cdk-phosphorylation site and the recognition/binding site for the cdk/cyclin complex, as for efficiently phosphorylating $\mathrm{pRb}$. $^{8}$ Moreover, in a functional test of myogenic conversion, cdk9/cyclin T2a was able to enhance the conversion of fibroblasts into myotubes by ectopic expression of MyoDsp1 and MyoDsp3, like MyoDwt, but failed to increase the activity of MyoDsp2 and MyoDsp $(1+2)$, which exhibited, again, a reduced myogenic potential when coexpressed with cdk9/cyclin T2a (Figure 1d).

These results establish that the enhancement of MyoD function by cdk9/cyclin T2a requires the presence of serine 37, a well-conserved residue in the MyoD protein of mammals (see Figure 1b). The normal basal activity of the MyoD serine 37 mutant (MyoDsp2) ${ }^{5}$ would appear in conflict with the proposed essential function of this serine in receiving an activator signal during muscle differentiation. The complex regulation of MyoD by a variety of signals and the autoactivatory network of muscle regulatory factors during myogenic differentiation may, at least partially, explain these data. Even when deficient in receiving one of the positive signals, MyoD can still stimulate a basal level of transcription, which may be enough to activate other myogenic regulatory factors, which can in turn activate the myogenic program by a parallel cdk9-independent pathway. This explanation is supported by the extremely high redundancy of activation of myogenic transcription in cell culture and in vivo models. ${ }^{9,10}$ Moreover, we cannot rule out that fibroblasts transiently overexpressing MyoD could be deficient for some muscle-specific proteins or signaling pathways, and ultimately that enforced expression of MyoD could be able to induce trans-differentiation, but not completely recapitulate the myogenic program. The hypothesis that serine 37 phosphorylation could be involved in protein-protein interactions might be suggested by an analogy to the proposed role for MyoD serine 5 in the recruitment of PCAF and HDAC1. ${ }^{11}$ During the preparation of this manuscript, lankova et $a^{12}$ reported that PPAR $\gamma$ recruits cdk9 complex on specific promoters to activate transcription and stimulate adipogenesis. In accordance with our model, they showed that cdk9 phosphorylates the adipogenic master regulator at serine 112 to transactivate PPAR $\gamma$-mediated gene expression. Furthermore, cdk9 can also phosphorylate p53 on serine residues 33,315 and $392,{ }^{13}$ confirming that the kinase complex could recognize signaldependent transcription factors as additional substrates besides the CTD of RNApollI. ${ }^{4}$

As the activity of MyoD is hampered in rhabdomyosarcoma cells, we decided to investigate the significance of cdk9mediated MyoD phosphorylation in the human cell line RD. For this purpose, we immunoprecipitated endogenous cdk9 complexes from cells induced to differentiate. Surprisingly, in tumor cells, cdk9 fails to phosphorylate MyoD (Figure 1a), although RD cells showed even upregulation of both cdk9 and cyclin T2 when compared to C2C12 myoblasts (Supplementary Information, B). Evaluation of immunoprecipitated complexes demonstrated that the antibody could recognize endogenous cdk9 protein, excluding conformational change of the kinase in cancer cells, and that the amount of precipitated protein was comparable between $\mathrm{C} 2 \mathrm{C} 12$ and RD cells (Supplementary Information, C, upper panel). Further characterization showed that the interaction between cdk9 and cyclin T2 was maintained or even increased, if compared to normal cells (Supplementary Information, C, lower panel). The paradoxical increase in the protein levels of cdk9/cyclin T2 complex in cancer cells resembled those of some tumor suppressor proteins, such as p53, whose overexpression often represents a hallmark of gene mutation. ${ }^{14}$ We explored this possibility by direct sequencing of the DNA coding sequence of cdk9 and cyclin T2 in RD cells, but we failed to detect any mutation. As endogenous cdk9 kinase activity appeared to be blocked, we verified if enforced expression of cdk9/cyclin T2a could rescue the function of the complex, but we failed to reach significant values of transcription activation even in the presence of overexpressed MyoD (Supplementary Information, D and E).

Our results seem to suggest that a signaling cascade targeting cdk9 can be inactive in RD cells. Recently, it was reported that $R D$ cells were defective in the MKK6/p38 pathway. ${ }^{15}$ Moreover, pharmacological blockade of the p38 pathway reduced the phosphorylation of the CTD of the RNApolll on muscle-specific regulatory regions, ${ }^{16}$ at residues targeted by cdk9 during transcriptional elongation. ${ }^{17}$ To test whether the signal propagated by $\mathrm{p} 38$ could affect cdk9 activity in muscle cells, we employed two $\mathrm{C} 2 \mathrm{C} 12$ polyclones carrying a chromatin-integrated MyoD-responsive luciferase reporter controlled, respectively, by the myogenin promoter (myogenin-luc) and the MCK enhancer (MCK-luc). Overexpression of the constitutively active form of the MKK6 kinase (MKK6EE) is able to strongly coactivate MyoD, inducing even endogenous muscle-specific genes, ${ }^{15,16,18}$ but when co-transfected with the dominant-negative form of cdk9 (cdk9dn), it failed to do so (Figure 1e and Supplementary Information, F). Significantly, pharmacological blockade of p38 using the specific inhibitor SB203580 abrogated MyoD-dependent transcription, even in the presence of overexpressed cdk9/cyclin T2a complex (Figure 1e and Supplementary Information, F). These data suggest a functional relationship between cdk9/cyclin T2a and the p38 pathway. We recently described that p38 signaling influences MyoDmediated cofactor recruitment on the chromatin of musclespecific genes, ${ }^{16}$ and observed that the cok9/cyclin T2 complex occupies these regions during the differentiation program. ${ }^{17}$ To evaluate whether p38 signaling is able to affect 
cdk9/cyclin T2 localization on the chromatin, we analyzed the presence of cdk9 and cyclin T2 on the MCK enhancer. We found that even if the binding of cdk9 to the DNA was unaltered, that of cyclin T2 depended, at least to a significant degree, on p38 signaling (Figure 1f). These findings suggest the hypothesis that a perturbation of the p38 pathway may abrogate the activity of the cdk9/cyclin T2 complex, probably by affecting the stable recruitment of cyclin T2 at the chromatin level. Upon MKK6-dependent enzymatic activation, p38 localizes on muscle-gene regulatory regions promoting the phosphorylation-dependent recruitment of SWI/SNF, chromatin remodeling and hyperphosphorylation of the CTD of RNApolll at these elements. ${ }^{16}$ At least one out of these events could be required for the stabilization of cyclin T2 binding to the DNA. Moreover, as both cdk9 and cyclin T2 can independently associate with MyoD in vitro, ${ }^{3}$ it is possible that one or more p38-dependent post-translational modifications imparted to the components of the MyoD transcriptional complex could influence the stable recruitment of the cyclin, even if cdk9 is retained on the chromatin.

At present, we cannot exclude that other mechanisms might be responsible for cdk9/cyclin T2 activation in muscle cells, and we believe it will be important to clarify whether the cdk9/ cyclin T2 complex is a direct target of the p38 pathway. Moreover, further studies are awaited to elucidate the significance of cdk9-dependent MyoD phosphorylation in normal and tumor muscle cells in vivo. We acknowledge that previous studies examined the phosphorylation pattern of MyoD without finding a major regulatory function for serine 37. 5 However, the phosphotryptic map analyses were performed only in proliferating myoblasts, and when the transcriptional activity of the MyoDsp2 mutant was tested in differentiating conditions, the authors merely employed luciferase reporter activity assays in fibroblasts. ${ }^{5}$ The actual modality of regulation of bHLH-dependent transcription by phosphorylation remains controversial, as numerous studies have reported discrepancies about the role of different kinases on the regulation of distinct bHLH factors. ${ }^{1}$ For example, phosphopeptide analysis of MyoD in myoblasts versus myotubes revealed the presence of a differentiationinduced spot, which corresponds to serine 5 phosphorylation. ${ }^{1}$ This phosphorylation is abrogated by inactivation of p38 and is ectopically produced in myoblasts by enforced activation of p38 by MKK6EE. ${ }^{1}$ Mutation of this residue eliminated the differentiation-induced serine 5 phosphorylation in myotubes, but does not significantly change the activity of MyoD in fibroblasts. ${ }^{1}$ Thus, although closely associated with the differentiation process and the activation of p38, the function for this phosphorylation site in myotubes is still unknown. Moreover, the proposed role for the concomitant phosphorylation at serine 5 and serine 200 by cdk 1 in myoblasts, resulting in the repression of MyoD activity and then decrease of its stability, ${ }^{11}$ seems to be controversial.

The presence of a large number of consensus sites for cdks, MAPKs and SAPKs in all the myogenic bHLH proteins would potentially implicate that multiple phosphorylation signals are required to coordinate muscle-specific transcrip- tion. Furthermore, it should be noted that a systematic mutation of all the proline-directed serines in MyoD did not produce any change of function in fibroblasts, except for serine 200. This scenario shows a complex regulation of MRFs by phosphorylation, and could explain why only cooverexpression of MyoDsp2 with the cdk9/cyclin T2a complex can demonstrate the role of serine 37 in MyoD transactivation. Similarly, serine 112 of PPAR $\gamma$ has been reported to be phosphorylated by MAPK, cdk7 and recently by $\operatorname{cdk} 9 ;^{12}$ however, the effects of this covalent modification are so far inconsistent since phosphorylation at the same residue by different kinases results in either activation or repression.

In conclusion, our results establish that cdk9/cyclin T2amediated coactivation of MyoD depends on serine 37 phosphorylation. The abrogation of this enzymatic modification in the human RD cell line suggests the hypothesis that the p38 pathway could represent the upstream cascade signaling to cdk9/cyclin T2 complex in muscle cells. The reported functional and biochemical links between p53, cdk9 ${ }^{13}$ and p38, ${ }^{19}$ together with the well-known role of these proteins in rhabdomyosarcoma formation, ${ }^{15,20}$ corroborate this hypothesis.

Acknowledgements. We thank Dr. Puri, Dr. Sartorelli and Dr. Harrington for kindly providing reagents, to Dr. Bagella, Dr. Stiegler and Dr. Bellan for their helpful collaboration, to Dr. Guanti, Dr. Resta and Dr. Stella for critical reading of the manuscript and to Marie Basso for her editorial assistance. Dr. Simone is supported by 'Scuola di Specializzazione in Genetica Medica' from the University of Bari.

\section{Simone ${ }^{*, 1,2}$ and A Giordano ${ }^{*, 1}$}

${ }^{1}$ Department of Biology, Sbarro Institute for Cancer Research and Molecular Medicine, Center for Biotechnology, College of Science and Technology, Temple University, 1900 N. 12th Street, Bio Life Sciences Building, Room 333, Philadelphia, PA 19122, USA

2 Department of Biomedicine in Childhood, Division of Medical Genetics, University of Bari, Bari 70124, Italy

* Corresponding authors: C Simone or A Giordano, Department of Biology, Sbarro Institute for Cancer Research and Molecular Medicine, Center for Biotechnology, College of Science and Technology, Temple University, 1900 N. 12th Street, Bio Life Sciences Building, Room 333, Philadelphia, PA 19122, USA. Tel: + 39-0805478391 or +215-204-9520; Fax: +39-0805478269 or + 215-204-9519; E-mails: csimone@ @emple.edu or giordano@temple.edu

1. Puri PL, Sartorelli V (2000) J. Cell. Physiol. 85: 155-173.

2. Sartorelli V, Puri PL (2001) Front. Biosci. 6: D1024-47.

3. Simone $C$ et al. (2002) Oncogene 21: 4137-4148.

4. Simone C, Giordano A (2001) Front. Biosci. 6: D1073-D1082.

5. Song A et al. (1998) Mol. Cell. Biol. 18: 4994-4999.

6. Kitzmann M et al. (1999) Mol. Cell. Biol. 19: 3167-3176.

7. Tintignac LA et al. (2000) Exp. Cell Res. 259: 300-307.

8. Simone $C$ et al. (2002) Oncogene 21: 4158-4165.

9. Lassar A, Munsterberg A (1994) Curr. Opin. Cell Biol. 6: 432-442.

10. Molkentin JD, Olson EN (1996) Proc. Natt. Acad. Sci. USA 93: 9366-9373.

11. Tintignac LA et al. (2004) Mol. Cell. Biol. 24: 1809-1821.

12. lankova I et al. (2006) Mol. Endocrinol 20: 1494-1505.

13. Radhakrishnan SK, Gartel AL (2006) Cell Cycle 5: 519-521.

14. Chang SC et al. (2005) Int. J. Oncol. 26: 65-75.

15. Puri PL et al. (2000) Genes Dev. 14: 574-584.

16. Simone $C$ et al. (2004) Nat. Genet. 36: 738-743.

17. Giacinti $C$ et al. (2006) J. Cell. Physiol. 206: 807-813.

18. Wu Z et al. (2000) Mol. Cell. Biol. 20: 1809-1821.

19. Sanchez-Prieto R et al. (2000) Cancer Res. 60: 2464-2472.

20. Merlino G, Helman LJ (1999) Oncogene 18: 5340-5348. 